\title{
NEGATIVE DEFINITE AND SCHOENBERG FUNCTIONS ON COMMUTATIVE HYPERGROUPS
}

\author{
WALTER R. BLOOM ${ }^{\varpi}$ and PAUL RESSEL
}

(Received 22 April 2003; revised 24 March 2004)

Communicated by A. H. Dooley

\begin{abstract}
In this paper we investigate when negative definite functions on commutative hypergroups satisfy the Schoenberg criterion.

2000 Mathematics subject classification: primary 43A62; secondary 43A10, 44A60.

Keywords and phrases: hypergroup, positive definite, negative definite, Sturm-Liouville, Schoenberg, quadratic form.

About sixty years ago Schoenberg contributed a range of fundamental results to the theory of positive definite and related functions which were subsequently generalized in many directions. One basic observation was that a function $\psi$ is negative definite if and only if $\exp (-t \psi)$ is positive definite for each $t>0$. While this result holds for all semigroups (in fact it is true for arbitrary negative definite kernels) it is not clear how to prove the 'only if' part for hypergroups since the usual techniques do not apply (the 'if' part always holds provided that $\operatorname{Re} \psi$ is locally lower bounded). The problem is that except when $x$ or $y$ belong to the maximal subgroup of the hypergroup $\exp (-t \psi(x * y))$ and $\exp (-t \psi)(x * y)$ are usually not equal so that other methods have to be used to overcome this. However no counterexample is known so far.

In this paper we closely analyze the negative definite functions on commutative hypergroups, in particular the Sturm-Liouville hypergroup structures on the halfline $\mathbb{R}_{+}$. This is achieved through a study of the Lévy-Khinchin representation with the key component being the non-negative quadratic forms. It should be pointed out that since $\mathbb{R}_{+}$carries the identity involution the quadratic forms are none other than the additive functions on these spaces.
\end{abstract}

Part of the work for this paper was carried out while the first author was visiting the University of Eichstätt.

(C) 2005 Australian Mathematical Society $1446-7887 / 05 \$ A 2.00+0.00$ 
As it turns out the non-negative additive functions on $\mathbb{R}_{+}$have been completely characterized (see $[2,7.3 .1]$ ) but they appear not to have a simple form. However we do have a reasonable catalogue of negative definite functions on many Sturm-Liouville hypergroups including the well-known Bessel-Kingman, Naimark, cosh and square hypergroups.

The paper is divided into two sections. We commence by introducing positive definite and negative definite functions together with quadratic forms on hypergroups, presenting the Lévy-Khinchin representation for negative definite functions with lower bounded real part, and then introduce Schoenberg functions. In the second section we consider the problem of showing that negative definite functions on $\mathbb{R}_{+}$satisfy a strong version of negative definiteness (that is, are Schoenberg).

Unless otherwise stated the notation will be that of [2] which is our main reference for harmonic analysis on these spaces.

\section{Positive and negative definite functions on hypergroups}

Let $(K, *)$ be a commutative hypergroup with neutral element $e$, involution ${ }^{-}$and Haar measure $\omega$. A locally bounded measurable function $\chi: K \rightarrow \mathbb{C}$ is called a semicharacter if

(i) $\chi(e)=1$,

(ii) $\chi(x * y)=\chi(x) \chi(y)$ for all $x, y \in K$,

(iii) $\chi\left(x^{-}\right)=\overline{\chi(x)}$ for all $x \in K$.

Every bounded semicharacter is called a character. If the character is not locally null then (see [2, Proposition 1.4.33]) it must be continuous. The dual $K^{\wedge}$ of $K$ is just the set of continuous characters with the compact-open topology in which case $K^{\wedge}$ must be locally compact. In this paper we will be concerned with continuous characters on hypergroups.

A locally bounded measurable function $\phi: K \rightarrow \mathbb{C}$ is said to be positive definite if

$$
\sum_{i=1}^{n} \sum_{j=1}^{n} c_{i} \bar{c}_{j} \phi\left(x_{i} * x_{j}^{-}\right) \geq 0
$$

for all choices of $x_{1}, x_{2}, \ldots, x_{n} \in K, c_{1}, c_{2}, \ldots, c_{n} \in \mathbb{C}$ and $n \in \mathbb{N}$. A locally bounded measurable function $\psi: K \rightarrow \mathbb{C}$ is said to be negative definite if $\psi\left(x^{-}\right)=\overline{\psi(x)}$ and

$$
\sum_{i=1}^{n} \sum_{j=1}^{n} c_{i} \bar{c}_{j} \psi\left(x_{i} * x_{j}^{-}\right) \leq 0
$$

whenever the $c_{1}, c_{2}, \ldots, c_{n}$ further satisfy $\sum_{i=1}^{n} c_{i}=0$. 
A range of properties of positive definite and negative definite functions can be found in [2, Chapter 4]. In particular, it should be noted that

(1.1) if $\psi$ is negative definite then $\psi+c$ is negative definite for all $c \in \mathbb{R}$, if $\phi$ is positive definite then $\phi(e)-\phi$ is negative definite.

We denote by $P(K)$ the space of bounded continuous positive definite functions on $K$ and by $N(K)$ the space of continuous negative definite functions $\psi$ with lower bounded real part (in which case $\operatorname{Re}(\psi) \geq \psi(e)$ ). Observe that $\chi \in P(K)$ for all $\chi \in K^{\wedge}$ so that by (1.2), $1-\chi \in N(K)$.

A locally bounded measurable function $q$ is called a quadratic form if

$$
q(x * y)+q\left(x * y^{-}\right)=2 q(x)+2 q(y)
$$

for all $x, y \in K$ and additive if $q(x * y)=q(x)+q(y)$ for all $x, y \in K$. In the case that $K$ is hermitian, that is when $K$ carries the identity involution, then every quadratic form is an additive function and every negative definite function is real.

We have the following extension of [2, Theorem 1.4.35].

PROPOSITION 1.1. Every quadratic form $q$ on a (commutative) hypergroup $K$ is continuous everywhere.

Proof. Choose $f \in C_{c}(K)$ satisfying $\int_{K} f d \omega=1$. Now using [2, Theorem 1.3.21]

$$
\begin{aligned}
\int_{K} f\left(x^{-} * y\right)\left(q(y)+q\left(y^{-}\right)\right) \omega(d y) & =\int_{K} f(y)\left(q(x * y)+q\left(x * y^{-}\right)\right) \omega(d y) \\
& =\int_{K} f(y)(2 q(x)+2 q(y)) \omega(d y) \\
& =2 q(x)+2 \int_{K} f(y) q(y) \omega(d y)
\end{aligned}
$$

and $q \in C(K)$ by [2, Proposition 1.4.27].

For hypergroups we have that if $\exp (-t \psi)$ is positive definite for all $t>0$ then $\exp (-t \psi(e))-\exp (-t \psi)$ is negative definite and provided $\operatorname{Re} \psi$ is locally lower bounded

$$
\lim _{t \rightarrow 0} \frac{\exp (-t \psi(e))-\exp (-t \psi)}{t}=\psi-\psi(e)
$$

is also negative definite in which case so is $\psi$. For abelian semigroups the converse statement also holds. However this result is not available for hypergroups which 
hinges on deciding whether $\exp (-\psi)$ is positive definite (which is only known for bounded negative definite $\psi$ or more generally for those $\psi$ which are the uniform limits on compact sets of bounded negative definite functions; for a discussion of these and related results see [4, Corollary 3.6 and Remark 3.8]).

A key result in the study of negative definite functions on hypergroups is the following Lévy-Khinchin representation (see [2, Theorem 4.5.2]).

THEOREM 1.2. Every real-valued and lower bounded $\psi \in N(K)$ has the representation

$$
\psi(x)=\psi(e)+q(x)+\int_{K^{\wedge} \backslash(1)}(1-\operatorname{Re}(\chi(x))) \eta(d \chi)
$$

for all $x \in K$ where $q$ is a non-negative quadratic form on $K$ and $\eta \in M_{+}\left(K^{\wedge} \backslash\{1\}\right)$. Both $q$ and the integral part $\psi-\psi(e)-q$ belong to $N(K)$ and the pair $(q, \eta)$ is uniquely determined by $\psi$ with $q$ being given by

$$
q(x)=\lim _{n \rightarrow \infty} \frac{\psi\left(x^{* n}\right)}{n^{2}}+\lim _{n \rightarrow \infty} \frac{\psi\left((x * x)^{* n}\right)}{2 n} .
$$

It is worth spending a moment analyzing the Lévy-Khinchin theorem. First the Lévy measure $\eta$ is usually not bounded and the only bounded quadratic form $q$ is the function identically zero. Secondly $x \mapsto \operatorname{Re}(\chi(x))$ is positive definite so that $1-\operatorname{Re}(\chi(x))$ is negative definite. In general, it is not true that $\psi(e)-\psi$ is positive definite when $\psi$ is negative definite. However if the product of characters is positive definite (which is necessary for $K^{\wedge}$ to be a so-called weak hypergroup; see [3, Proposition 1]), in which case the product of continuous positive definite functions is again positive definite, then the power series expansion of the exponential shows that

$$
\exp (-t \psi)=\exp (-t \psi(e)) \exp (t(\psi(e)-\psi)) \in P(K)
$$

for all $t>0$ whenever $\psi(e)-\psi$ is positive definite.

Finally it should be observed that there exist unbounded negative definite functions with zero quadratic form part. For example on the group $\mathbb{R}$ (which is also a hypergroup with the usual involution), $x \mapsto x^{2}$ is negative definite and hence so is $x \mapsto|x|^{\alpha}$, $0<\alpha<2$. The uniquely determined quadratic form part is easily seen to be zero (see (1.3)) and indeed there exists unique $\eta_{\alpha} \in M_{+}\left(K^{\wedge} \backslash\{\mathbb{1}\}\right)$ with

$$
|x|^{\alpha}=\int_{\mathbb{R}}(1-\exp (-i y x)) \eta_{\alpha}(d y)
$$

(all non-negative quadratic forms on the group $\mathbb{R}$ are given by $x \mapsto a x^{2}$ with $a \geq 0$ ). 
1.1. Schoenberg functions A continuous function $\psi$ on $K$ is called a Schoenberg function if $\exp (-t \psi) \in P(K)$ for all $t>0$, in which case $\psi$ is necessarily negative definite. We write $\Psi(K)$ for the set of all Schoenberg functions on $K$.

As we have already observed every bounded continuous negative definite function is Schoenberg and hence the uniform limit on compact sets of bounded negative definite functions is again Schoenberg. We can use the Lévy-Khinchin representation to identify further Schoenberg functions.

THEOREM 1.3. If $\psi \in N(K)$ is real-valued and has zero quadratic part then $\psi \in \Psi(K)$.

PROOF. We apply Theorem 1.2 to obtain immediately

$$
\psi(x)=\psi(e)+\int_{K^{\wedge} \backslash\{1\}}(1-\operatorname{Re}(\chi(x))) \eta(d \chi),
$$

where $\eta \in M_{+}\left(K^{\wedge} \backslash\{\mathbb{1}\}\right)$. The result will now follow by appealing to [2, Theorem 4.4.16] once we know that $\psi(x)-\psi(e)$ satisfies the following version of negative definiteness: Denote the Fourier transform of $\mu \in M^{b}(K)$ (the space of bounded complex-valued Radon measures on $K)$ by $\hat{\mu}(\chi):=\int_{K} \chi d \mu$. If $\mu \in M^{b}(K)$ has compact support $\hat{\mu} \geq 0$ and $\int_{K} d \mu=0$ then

$$
\begin{aligned}
\int_{K}(\psi(x)-\psi(e)) \mu(d x) & =\int_{K} \int_{K^{\wedge} \backslash\{1\}}(1-\operatorname{Re}(\chi(x))) \eta(d \chi) \mu(d x) \\
& =\int_{K^{\wedge} \backslash\{1\}}\left[\int_{K}(1-\operatorname{Re}(\chi(x))) \mu(d x)\right] \eta(d \chi) \\
& =-\int_{K^{\wedge} \backslash\{1\}}\left[\int_{K} \operatorname{Re}(\chi(x)) \mu(d x)\right] \eta(d \chi) \\
& =-\frac{1}{2} \int_{K^{\wedge} \backslash\{1\}}(\hat{\mu}(\bar{\chi})+\hat{\mu}(\chi)) \eta(d \chi) \\
& \leq 0
\end{aligned}
$$

as required.

In the classical case the composition of a Bernstein function (that is, a continuous non-negative negative definite function on the semigroup $\left.\left(\mathbb{R}_{+},+\right)\right)$and a non-negative negative definite function is again negative definite. While this might be true for hypergroups we only have an analogue for Schoenberg functions. We first consider the following type of convergence.

DEFINITION. A sequence of functions $\left(g_{n}\right)$ is termed locally bounded convergent with limit $g$ if $\sup \left\{g_{n}(x) \mid: n=1,2, \ldots, x \in C\right\}$ is finite for each compact set $C \subset K$ and $\lim _{n \rightarrow \infty} g_{n}=g$ pointwise on $K$. 
It is easy to see that local uniform convergence implies local bounded convergence.

For the rest of this section we will assume that products of characters are positive definite. We now present two easy lemmas.

LEMMA 1.4. Each of the sets of continuous positive definite functions and continuous negative definite functions on $K$ is closed under sequential local bounded convergence.

LEMMA 1.5. The set $\Psi(K)$ of all Schoenberg functions on $K$ is a convex cone closed under sequential local bounded convergence.

PROPOSITION 1.6. If $\psi \in \Psi(K)$ and $t>0$ then $1-\exp (-t \psi) \in \Psi(K)$.

PROOF. Put $\varphi_{t}:=\exp (-t \psi)$ and let $s>0$. Then

$$
\exp \{-s[1-\exp (-t \psi)]\}=e^{-s} \sum_{n=0}^{\infty} \frac{s^{n}}{n !} \varphi_{t}^{n}
$$

is again positive definite.

COROLLARY 1.7. For every Bernstein function $f: \mathbb{R}_{+} \rightarrow \mathbb{R}_{+}$the composition $f \circ \psi$ with a non-negative Schoenberg function $\psi$ is again a Schoenberg function.

Proof. It needs only to be observed that Bernstein functions are of the form

$$
f(t)=a+b t+\int_{0}^{\infty}\left(1-e^{-t x}\right) \mu(d x)
$$

where $\mu \in M_{+}(] 0, \infty[)$ satisfies $\int_{0}^{\infty}(x /(1+x)) \mu(d x)<\infty$ and $a, b \geq 0$ are constants (see [1, Remark, page 114]) and then apply Proposition 1.6.

To apply Corollary 1.7 we need a catalogue of Bernstein functions. Two wellknown ones give immediately that for $0<s<1$, if $\psi \in \Psi(K)$ then $\psi^{s} \in \Psi(K)$ and likewise $\log (1+\psi) \in \Psi(K)$. Further examples can be easily obtained from the criterion that a continuous function $f: \mathbb{R}_{+} \rightarrow \mathbb{R}_{+}$is Bernstein if and only if $f \in C^{\infty}(] 0, \infty[)$ and $(-1)^{n} f^{(n+1)} \geq 0$ for all $n=0,1, \ldots$ (see [1, page 141]).

In Theorem 1.3 the integral part

$$
\int_{K^{\wedge} \backslash(1)}(1-\operatorname{Re}(\chi(x))) \eta(d \chi)
$$

has been shown to be a Schoenberg function. Thus to prove that a general lower bounded negative definite function is Schoenberg we need only consider its quadratic form part. 


\section{Quadratic forms on commutative hypergroups}

Additive functions on a Sturm-Liouville hypergroup $\mathbb{R}_{+}$are given as in $[2$, Sections 7.2-7.3] as first and second order moment functions. In particular, (see [2, Example 7.3.1]) the canonical first order moment function

$$
m_{1}(x):=2 \rho \int_{0}^{x} \int_{0}^{y} \frac{A(z)}{A(y)} d z d y
$$

gives an additive function on $\mathbb{R}_{+}$and to within a multiplicative constant this additive function is unique. Here $A$ is the Sturm-Liouville function defining the hypergroup and $\rho:=(1 / 2) \lim _{x \rightarrow \infty} A^{\prime}(x) / A(x)$ is its index. If $\rho=0$, so that $m_{1}=0$, then the canonical second order moment function

$$
m_{2}(x):=2 \int_{0}^{x} \int_{0}^{y} \frac{A(z)}{A(y)} d z d y
$$

gives an additive function on $\mathbb{R}_{+}$and once again to within a multiplicative constant this additive function is unique.

For any commutative hypergroup $K$ let $\chi_{t} \in K^{\wedge}$ for all $t \in[0,1]$. Suppose that the mapping $t \rightarrow \chi_{t}$ is differentiable on $[0,1]$ with $\chi_{0}=1_{K}$ and write

$$
m_{1}=-\left.\frac{\partial \chi_{t}}{\partial t}\right|_{t=0}
$$

(see [2, page 469]), where we assume that the limit defining the derivative holds locally boundedly on $K$. Then $m_{1}$ is additive (hence a quadratic form) on $K$ which we can see from

$$
\begin{aligned}
m_{1}(x * y) & =-\left.\int_{K} \frac{\partial \chi_{t}}{\partial t}\right|_{t=0} d\left(\varepsilon_{x} * \varepsilon_{y}\right)=-\left.\frac{\partial}{\partial t} \int_{K} \chi_{t} d\left(\varepsilon_{x} * \varepsilon_{y}\right)\right|_{t=0} \\
& =-\left.\frac{\partial}{\partial t} \chi_{t}(x) \chi_{t}(y)\right|_{t=0}=m_{1}(x) \chi_{0}(y)+\chi_{0}(x) m_{1}(y) \\
& =m_{1}(x)+m_{1}(y) .
\end{aligned}
$$

Now from Theorem 1.3 we see that each $1-\chi_{t} \in \Psi(K)$ and

$$
m_{1}=-\left.\frac{\partial \chi_{t}}{\partial t}\right|_{t=0}=\lim _{t \downarrow 0} \frac{1-\chi_{t}}{t} \in \Psi(K)
$$

as the limit is locally bounded on $K$. If $m_{1}=0$ (which by $[2,7.3 .1$ and Proposition 3.5.55] holds for Sturm-Liouville hypergroups of sub-exponential growth) then 
we consider the second moment

$$
\begin{aligned}
m_{2} & =-\left.\frac{\partial^{2} \chi_{t}}{\partial t^{2}}\right|_{t=0}=-\left.\frac{\partial}{\partial t}\left(\lim _{s \rightarrow 0} \frac{\chi_{t+s}-\chi_{t}}{s}\right)\right|_{t=0} \\
& =-\left.\lim _{h \rightarrow 0} \frac{\lim _{s \rightarrow 0}\left(\chi_{t+s+h}-\chi_{t+h}\right) / s-\lim _{s \rightarrow 0}\left(\chi_{t+s}-\chi_{t}\right) / s}{h}\right|_{t=0} \\
& =-\left.\lim _{h \rightarrow 0} \lim _{s \rightarrow 0} \frac{\chi_{t+s+h}-\chi_{t+h}-\chi_{t+s}+\chi_{t}}{s h}\right|_{t=0} \\
& =-\lim _{h \rightarrow 0} \lim _{s \rightarrow 0} \frac{\chi_{s+h}-\chi_{h}-\chi_{s}+1}{s h}=\lim _{t \downarrow 0} \lim _{s \downarrow 0} \frac{\chi_{t}-\chi_{s+t}}{s t}
\end{aligned}
$$

since $m_{1}=\lim _{s \rightarrow 0}\left(1-\chi_{s}\right) / s=0$. Furthermore (as shown above for $m_{1}$ ) $m_{2}$ is easily seen to be additive but it is not obvious that this limit function should belong to $\Psi(K)$.

2.1. Moment functions and semicharacters on Sturm-Liouville hypergroups of exponential growth $(\rho>0)$ By [2, Theorem 3.5.58] we have, for each $x \in \mathbb{R}_{+}$, the existence of $v_{x} \in M^{1}([-x, x])$ satisfying the Laplace representation

$$
\phi_{\lambda}(x)=\int_{-x}^{x} e^{-(\rho+i \lambda) t} v_{x}(d t)
$$

for all $\lambda \in \mathbb{C}$, where $\left\{\phi_{\lambda}: \lambda \in \mathbb{C}\right\}$ denotes the set of semicharacters of $K$. We observe from $[2,7.2 .2]$ that

$$
m_{1}(x)=\left.\frac{\partial}{\partial \sigma} \phi_{i(\rho+\sigma)}(x)\right|_{\sigma=0}
$$

Now appealing to (2.1) we have

$$
\phi_{i(\rho+\sigma)}(x)=\int_{-x}^{x} e^{-(\rho+i i(\rho+\sigma)) t} v_{x}(d t)=\int_{-x}^{x} e^{\sigma t} v_{x}(d t)
$$

and

$$
m_{1}(x)=\left.\frac{\partial}{\partial \sigma} \phi_{i(\rho+\sigma)}(x)\right|_{\sigma=0}=\left.\int_{-x}^{x} \frac{\partial}{\partial \sigma} e^{\sigma t}\right|_{\sigma=0} v_{x}(d t)=\int_{-x}^{x} t v_{x}(d t) .
$$

We also observe that

$$
m_{1}(x)=2 \rho \int_{0}^{x} \int_{0}^{y} \frac{A(z)}{A(y)} d z d y
$$

is an increasing function with $m_{1}(0)=0$. 
Now for $x \in[0, a]$ and $\sigma \in(0,1)$, consider (recall that $\phi_{i \rho}=1$ )

$$
\begin{aligned}
\left|\frac{\phi_{i \rho}(x)-\phi_{i(\rho-\sigma)}(x)}{\sigma}\right| & =\left|\frac{1-\int_{-x}^{x} e^{-\sigma t} v_{x}(d t)}{\sigma}\right| \leq \int_{-x}^{x}\left|\frac{1-e^{-\sigma t}}{\sigma t}\right| t v_{x}(d t) \\
& \leq \frac{1+e^{a}}{a} \int_{-x}^{x}|t| v_{x}(d t) \leq 1+e^{a}
\end{aligned}
$$

since $\left|\left(1-e^{-u}\right) / u\right| \leq\left(1+e^{a}\right) / a$ for all $u \in[-a, a]$ and this takes care of the local boundedness for Sturm-Liouville hypergroups of exponential growth.

\subsection{Moment functions on Sturm-Liouville hypergroups of exponential growth} $(\rho>0)$ are Schoenberg We have

$$
m_{1}=-\left.\frac{\partial \chi_{t}}{\partial t}\right|_{t=0},
$$

where $\chi_{t}=\phi_{i \rho(1-t)}$. If $\mu \in M^{b}\left(\mathbb{R}_{+}\right)$has compact support, $\hat{\mu} \geq 0$ and $\int_{K} d \mu=0$ then writing $f(t):=\hat{\mu}(i \rho(1-t))=\int_{\mathbb{R}_{+}} \chi_{t}(x) \mu(d x)$ we have (assuming that the limit defining the derivative holds locally boundedly on $K$ )

$$
-f^{\prime}(0)=-\left.\int_{\mathbb{R}_{+}} \frac{\partial \chi_{t}(x)}{\partial t}\right|_{t=0} \mu(d x)=\int_{\mathbb{R}_{+}} m_{1}(x) \mu(d x) .
$$

Now the conditions on $\hat{\mu}$ guarantee that $f^{\prime}(0) \geq 0$ (for if $f^{\prime}(0)<0$ then since $f(0)=\hat{\mu}(i \rho)=0$ we would have $f<0$ on some interval $(0, \delta)$ ) so that

$$
\int_{\mathbb{R}_{+}} m_{1}(x) \mu(d x) \leq 0
$$

and appealing to [2, Theorem 4.4.16] we see that $m_{1} \in \Psi(K)$.

The following Sturm-Liouville hypergroups are of exponential growth and hence all real-valued lower bounded continuous negative definite functions on these are Schoenberg.

(a) cosh hypergroup $([2,3.5 .72]) A(x)=\cosh ^{2} x, \rho=1, m_{1}(x)=x \tanh x$;

(b) Jacobi hypergroup of non-compact type ([2, 3.5.64])

$$
A(x)=\sinh ^{2 \alpha+1} x \cosh ^{2 \beta+1} x ;
$$

(c) Hyperbolic hypergroups ([2, 3.5.65]) $A(x)=\sinh ^{2 \alpha+1} x, \rho=2 \alpha+1$;

(d) Naimark hypergroup $([2,3.5 .65])$

$$
A(x)=\sinh ^{2} x, \quad \rho=2, \quad m_{1}(x)=x \operatorname{coth} x-1
$$


2.3. Moment functions and semicharacters on Sturm-Liouville hypergroups of sub-exponential growth $(\rho=0)$ Assuming that the limits defining both derivatives hold locally boundedly on $K$ we have

$$
m_{1}=-\left.\frac{\partial \chi_{t}}{\partial t}\right|_{t=0}=0 \quad \text { and } \quad m_{2}=-\left.\frac{\partial^{2} \chi_{t}}{\partial t^{2}}\right|_{t=0},
$$

where $\chi_{t}=\phi_{t}$. Again, if $\mu \in M^{b}\left(\mathbb{R}_{+}\right)$has compact support, $\hat{\mu} \geq 0$ and $\int_{K} d \mu=0$ then writing $f(t):=\hat{\mu}(t)=\int_{\mathbb{R}_{+}} \chi_{t}(x) \mu(d x)$ we have

$$
-f^{\prime}(0)=-\left.\int_{\mathbb{R}_{+}} \frac{\partial \chi_{t}(x)}{\partial t}\right|_{t=0} \mu(d x)=\int_{\mathbb{R}_{+}} m_{1}(x) \mu(d x)=0
$$

and

$$
-f^{\prime \prime}(0)=-\left.\int_{\mathbb{R}_{+}} \frac{\partial^{2} \chi_{t}(x)}{\partial t^{2}}\right|_{t=0} \mu(d x)=\int_{\mathbb{R}_{+}} m_{2}(x) \mu(d x) .
$$

As before the conditions on $\hat{\mu}$ guarantee that $f^{\prime \prime}(0) \geq 0$ so that we have

$$
\int_{\mathbb{R}_{+}} m_{2}(x) \mu(d x) \leq 0
$$

and once again appealing to [2, Theorem 4.4.16] we see that $m_{2} \in \Psi(K)$. But note that this deduction is made assuming a certain local boundedness of the limits defining the first and second order derivatives.

We have the following examples for which every real-valued lower bounded continuous negative definite function being Schoenberg can be shown directly.

(e) Bessel-Kingman hypergroup $\left(\mathbb{R}_{+}, *_{\alpha}\right)$

$$
\begin{aligned}
\alpha> & -1 / 2, \quad A(x)=x^{2 \alpha+1}, \quad \rho=0, \quad q(x)=x^{2}, \\
\varepsilon_{x} * \varepsilon_{y}= & \frac{\Gamma(\alpha+1)}{\Gamma(1 / 2) \Gamma(\alpha+1 / 2) 2^{2 \alpha-1}} \\
& \times \int_{|x-y|}^{x+y} \frac{\left[\left(z^{2}-(x-y)^{2}\right)\left((x+y)^{2}-z^{2}\right)\right]^{\alpha-1 / 2}}{(x y z)^{2 \alpha}} \varepsilon_{z} z^{2 \alpha+1} d z .
\end{aligned}
$$

A special case of the Bessel Kingman hypergroup is the motion hypergroup obtained by putting $\alpha=d / 2-1$, where $d$ is an integer with $d \geq 2$. In particular, taking $d=3$ we have the motion hypergroup.

(f) Motion hypergroup $\left(\mathbb{R}_{+}, *_{M}\right)$

$$
\varepsilon_{x} * \varepsilon_{y}=\frac{1}{2 x y} \int_{|x-y|}^{x+y} t \varepsilon_{t} d t, \quad A(x)=x^{2}, \quad \rho=0, \quad q(x)=x^{2} .
$$

Another example is obtained by letting $\alpha \rightarrow-1 / 2$ in which case we obtain the symmetric hypergroup. 
(g) Symmetric hypergroup $\left(\mathbb{R}_{+}, *_{2}\right)$

$$
\varepsilon_{x} * \varepsilon_{y}=\left(\varepsilon_{|x-y|}+\varepsilon_{x+y}\right) / 2, \quad A(x)=1, \quad \rho=0, \quad q(x)=x^{2} .
$$

We now show (Proposition 2.1 and Proposition 2.2 below) that for each of these hypergroups $x^{p}$ is a Schoenberg function for all $\left.\left.p \in\right] 0,2\right]$.

PROPOSITION 2.1. On the symmetric hypergroup $\left(\mathbb{R}_{+}, *_{2}\right)$ the function $\psi_{p}$ defined by $\psi_{p}(x)=x^{p}$ for $\left.\left.p \in\right] 0,2\right]$ is Schoenberg.

PROOF. Consider the kernel $\phi_{p, t}(x, y)=\exp \left(-t|x-y|^{p}\right)+\exp \left(-t(x+y)^{p}\right)$. Now

$$
\exp \left(-t|u|^{p}\right)=\int_{\mathbb{R}} \exp (i u \lambda) v_{p, t}(d \lambda)
$$

for some $v_{p, t} \in M_{+}^{1}(\mathbb{R})$ using Schoenberg's theorem for the real line and the fact that $|x|^{p}$ is negative definite on the group $\mathbb{B}$ for all $\left.\left.p \in\right] 0,2\right]$ together with Bochner's theorem. Thus

$$
\begin{aligned}
\phi_{p, t}(x, y) & =\int_{\mathbb{R}}[\exp (i(x-y) \lambda)+\exp (i(x+y) \lambda)] v_{p, t}(d \lambda) \\
& \left.\left.=\int_{\mathbb{R}}[\cos (x-y) \lambda)+\cos (x+y) \lambda\right)\right] v_{p, t}(d \lambda) \\
& =\int_{\mathbb{R}}[2 \cos x \lambda \cos y \lambda] v_{p, t}(d \lambda),
\end{aligned}
$$

where in the second equality we have used the fact that $\phi_{p, t}(x, y)$ is real valued. But this is just a positive mixture of positive definite kernels on $\mathbb{R}$ and hence $\phi_{p, t}(x, y)$ is a positive definite kernel on $\mathbb{R}$ and thus also on $\mathbb{R}_{+}$; this takes care of the result.

The above result is particularly surprising in view of the fact that it can be shown that the kernel $(x, y) \mapsto \exp \left(-(x+y)^{p}\right)$ is positive definite on $\left(\mathbb{R}_{+},+\right)$only for $p \in] 0,1]$ even though the $\operatorname{kernel}(x, y) \mapsto \exp \left(-|x-y|^{p}\right)$ is positive definite on $\mathbb{R}$ for all $p \in] 0,2]$. Indeed, to appreciate the difficulties that have been hidden consider the direct approach in which to show that $\exp \left(-\psi_{p}\right) \in P\left(\mathbb{R}_{+}, *_{2}\right)$ we would need to compute

$$
\sum_{i=1}^{n} \sum_{j=1}^{n} c_{i} c_{j} e^{-\psi_{p}}\left(x_{i} * x_{j}\right)=\sum_{i=1}^{n} \sum_{j=1}^{n} c_{i} c_{j}\left(e^{-\left|x_{i}-x_{j}\right|^{p}}+e^{-\left(x_{i}+x_{j}\right)^{p}}\right) / 2 .
$$

PROPOSITION 2.2. On the motion hypergroup $\left(\mathbb{R}_{+}, *_{M}\right)$ the function $\psi_{p}$ defined by $\psi_{p}(x)=x^{p}$ for $\left.\left.p \in\right] 0,2\right]$ is Schoenberg. 
PROOF. We first consider the case $p=2$. As in Proposition 2.1 consider the kernel

$$
\begin{aligned}
\phi_{t}(x, y) & =\frac{1}{2 x y} \int_{|x-y|}^{x+y} u \exp \left(-t u^{2}\right) d u \\
& =\frac{-1}{4 x y t}\left(\exp \left(-t(x+y)^{2}\right)-\exp \left(-t(x-y)^{2}\right)\right) \\
& =\frac{-1}{4 x y t} \int_{\mathbb{R}}[\exp (i(x+y) \lambda)-\exp (i(x-y) \lambda)] v_{2, t}(d \lambda) \\
& \left.\left.=\frac{-1}{4 x y t} \int_{\mathbb{R}}[\cos (x+y) \lambda)-\cos (x-y) \lambda\right)\right] v_{2, t}(d \lambda) \\
& =\frac{1}{2 x y t} \int_{\mathbb{R}}[\sin (x \lambda) \sin (y \lambda)] v_{2, t}(d \lambda),
\end{aligned}
$$

which again is a positive mixture of positive definite kernels on $\mathbb{R}$ and hence $\phi_{t}(x, y)$ is a positive definite kernel on $\mathbb{R}$ and thus also on $\mathbb{R}_{+}$. The argument in the proof of Proposition 2.1 gives that $\psi_{2} \in \Psi(K)$ and for the remaining values of $p$ we just apply Corollary 1.7 and the remarks immediately following it.

In discussions with the first author Michael Voit has pointed out that Proposition 2.1 and Proposition 2.2 when $p=2$ can also be deduced from [2, Example 7.3.18] which gives for every Bessel-Kingman hypergroup (including the limiting symmetric hypergroup) an explicit representation of the Gauss distribution $v_{t}$

$$
\hat{v}_{t}(\lambda):=\exp \left(-t \lambda^{2}\right)
$$

where $t>0$ and $\lambda \in \mathbb{R}_{+}$. This works because the Bessel-Kingman hypergroups are self-dual. In fact this representation can be used to give the following extension of the previous examples.

PROPOSITION 2.3. Let $\left(\mathbb{R}_{+}, *_{\alpha}\right)$ denote the Bessel Kingman hypergroup with parameter $\alpha \geq-1 / 2$ (this includes the symmetric case). Then the function $\psi_{p}$ defined by $\psi_{p}(x)=x^{p}$ for $\left.\left.p \in\right] 0,2\right]$ is Schoenberg.

Proof. As above we just need to show that $\psi_{2}$ is Schoenberg. But this follows immediately from (2.2) as we know that the Bessel Kingman hypergroup is self-dual; see $[2$, Theorem 3.6 .5$]$ and the remarks immediately preceding it.

2.4. Other hypergroups The results of the preceding subsections carry over to all hypergroups for which the first and second moment functions are given as at the beginning of Section 2 and the limits defining these hold locally boundedly on $K$. We have shown that the Sturm-Liouville hypergroups of exponential growth constitute one such class. 
We have also presented a range of examples of Sturm-Liouville hypergroups of subexponential growth for which every real-valued lower bounded continuous negative definite function is Schoenberg. There is one further well-known example of such hypergroups on the half-line that could be considered namely the square hypergroup $\left([2,3.5 .70], A(x)=(x+1)^{2}, \rho=0, q(x)=x^{2}(x+3) /(x+1)\right)$. In this case the corresponding quadratic form is Schoenberg as the defining double limit can be shown directly to be locally bounded.

\section{References}

[1] C. Berg, J. P. R. Christensen and P. Ressel, Harmonic analysis on semigroups. Theory of positive definite and related functions (Springer, New York, 1984).

[2] W. R. Bloom and H. Heyer, Harmonic analysis of probability measures on hypergroups (Walter de Gruyter, Berlin, 1995).

[3] R. Lasser, 'Orthogonal polynomials and hypergroups', Rend. Mat. 3 (1983), 185-209.

[4] M. Voit, 'Negative definite functions on commutative hypergroups', in: Probability measures on groups, IX (Oberwolfach 1988), Lecture Notes in Math. 1379 (Springer, Berlin, 1989) pp. 376-388.

Division of Science and Engineering Murdoch University

Perth, WA 6150

Australia

e-mail:w.bloom@murdoch.edu.au
Mathematisch-Geographische Fakultät

Katholische Universität Eichstätt

85072 Eichstätt

Germany

e-mail: paul.ressel@ku-eichstaett.de 
J. Aust. Math. Soc. 79 (2005) 